\title{
Comparison of coffee, tea and green tea consumption between Japanese with and without metabolic syndrome in a cross-sectional study
}

\author{
Nobuyuki Miyatake ${ }^{1}$, Noriko Sakano ${ }^{1}$, Takeyuki Numata ${ }^{2}$ \\ ${ }^{1}$ Department of Hygiene, Faculty of Medicine, Kagawa University, Kagawa, Japan \\ ${ }^{2}$ Okayama Southern Institute of Health, Okayama Health Foundation, Okayama, Japan \\ Email: miyarin@med.kagawa-u.ac.jp
}

Received 15 February 2012; revised 5 March 2012; accepted 27 March 2012

\begin{abstract}
There are some reports that coffee consumption improves insulin resistance. Therefore, we investigated the link between metabolic syndrome and coffee, tea and green tea consumption in Japanese. We used data of 150 men and 227 women who were not taking any medications, aged 22 - 74 years, in this cross-sectional investigation study. Habitual coffee, tea and green tea consumption was defined as drinking one or more cups of coffee, tea and green tea per day. The diagnosis of metabolic syndrome was based on the Japanese criteria. In subjects without medications, 34 men (22.7\%) and 10 women $(4.4 \%)$ were diagnosed with metabolic syndrome. Coffee and green tea consumption was weakly and positively correlated with age in women. Significant differences of coffee consumption between women with and without abdominal obesity, dyslipidemia and hypertension, tea consumption between men with and without dyslipidemia were noted after adjusting for age. However, there were no significant differences of other consumption between subjects with and without metabolic syndrome in both sexes. Among Japanese not taking medications, coffee, tea and green tea consumption was not clearly associated with metabolic syndrome in the Japanese population.
\end{abstract}

Keywords: Coffee Consumption; Metabolic Syndrome; Japanese

\section{INTRODUCTION}

Metabolic syndrome, characterized by abdominal obesity, has become public health challenge in Japan [1]. We previously reported that $30.7 \%$ of men and $3.6 \%$ of women were diagnosed as having metabolic syndrome [2]. Metabolic syndrome is closely linked to an increased risk for cardiovascular disease [3], proteinuria [4], the elevation of hepatic enzymes [5] and serum uric acid levels [6]. Therefore, the prevention and improvement of metabolic syndrome is urgently required.

Coffee, tea and green tea are common frequently consumed beverages. For example, 10.6 coffee cups per week are reported to be consumed [7] and about $50 \%$ of Japanese drinks coffee daily [8]. There some studies showed that habitual coffee consumption may improve insulin resistance and abdominal glucose metabolism [911]. However, the link between metabolic syndrome taking no medications and coffee, tea and green tea consumption in Japanese is still remains to be investigated.

In this study, we compared the coffee, tea and green tea consumption between Japanese with and without metabolic syndrome in a cross-sectional study.

\section{SUBJECTS AND METHODS}

\subsection{Subjects}

We used the data for 150 men and 227 women, aged 22 74 years, who met the following criteria, 1) they had wanted to change their lifestyle i.e. diet and exercise habits, and had received annual health checkups from April 2006 to December 2010 at Okayama Southern Institute of Health, Okayama Health Foundation, Japan, 2) they had received anthropometric measurements, fasting blood examination, and evaluation of coffee, tea and green tea consumption, 3) they had revived taking no medications such as diabetes, dyslipidemia, hypertension, infectious and/or neoplastic conditions, and 4) they had obtained written informed consent (Table 1).

The study was approved by the Ethics Committee of Okayama Health Foundation.

\subsection{Anthropometric and Body Composition Measurements}

The anthropometric parameters were evaluated by using 
Table 1. Clinical profiles of enrolled subjects.

\begin{tabular}{|c|c|c|c|c|c|c|}
\hline & \multicolumn{3}{|c|}{ Men } & \multicolumn{3}{|c|}{ Women } \\
\hline & Mean \pm SD & Minimum & Maximum & Mean \pm SD & Minimum & Maximum \\
\hline Number of subjects & 150 & & & 227 & & \\
\hline Age & $47.0 \pm 12.3$ & 22 & 74 & $43.5 \pm 14.8$ & 22 & 74 \\
\hline Height (cm) & $170.6 \pm 5.3$ & 156.5 & 184.2 & $157.2 \pm 4.9$ & 143.1 & 171.5 \\
\hline Body weight (kg) & $72.8 \pm 11.2$ & 50.0 & 125.5 & $55.9 \pm 8.6$ & 29.3 & 88.1 \\
\hline Body mass index $\left(\mathrm{kg} / \mathrm{m}^{2}\right)$ & $25.0 \pm 3.5$ & 15.9 & 38.4 & $22.6 \pm 3.2$ & 14.3 & 32.9 \\
\hline Abdominal circumference $(\mathrm{cm})$ & $87.0 \pm 9.6$ & 67.0 & 123.5 & $78.4 \pm 9.5$ & 58.5 & 111.1 \\
\hline Hip circumference (cm) & $96.0 \pm 6.5$ & 81.0 & 125.5 & $92.8 \pm 6.1$ & 70.0 & 118.0 \\
\hline Systolic blood pressure (mmHg) & $128.7 \pm 12.7$ & 94.0 & 183.0 & $116.9 \pm 15.6$ & 86.0 & 182.0 \\
\hline Diastolic blood pressure $(\mathrm{mmHg})$ & $78.6 \pm 10.0$ & 56.0 & 105.0 & $68.0 \pm 10.2$ & 46.0 & 106.0 \\
\hline Triglyceride (mg/dl) & $137.5 \pm 93.0$ & 24.0 & 670.0 & $87.6 \pm 52.3$ & 28.0 & 354.0 \\
\hline HDL cholesterol (mg/dl) & $57.2 \pm 14.8$ & 32.0 & 111.0 & $70.5 \pm 16.4$ & 22.0 & 144.0 \\
\hline Blood glucose (mg/dl) & $98.0 \pm 12.9$ & 67.0 & 164.0 & $91.8 \pm 10.9$ & 68.0 & 159.0 \\
\hline Coffee consumption (cup/week) & $10.1 \pm 9.1$ & 0 & 40 & $8.6 \pm 9.0$ & 0 & 50 \\
\hline Tea consumption (cup/week) & $1.5 \pm 4.1$ & 0 & 30 & $2.1 \pm 2.9$ & 0 & 15 \\
\hline Green tea consumption (cup/week) & $6.3 \pm 6.6$ & 0 & 40 & $7.4 \pm 8.6$ & 0 & 70 \\
\hline Number of subjects with exercise habits & $49(32.7 \%)$ & & & $43(18.9 \%)$ & & \\
\hline Number of subjects with smoking habits & $43(28.7 \%)$ & & & $14(6.2 \%)$ & & \\
\hline
\end{tabular}

the following respective parameters such as height, body weight, body mass index (BMI), abdominal circumference, and hip circumference. BMI was calculated by weight $/[\text { height }]^{2}\left(\mathrm{~kg} / \mathrm{m}^{2}\right)$. The abdominal circumference was measured at the umbilical level and the hip was measured at the widest circumference over the trochanter in standing subjects after normal expiration [1].

\subsection{Blood Pressure Measurements}

Each participant's blood pressure was measured after resting at least 15 minutes in the sitting position.

\subsection{Blood Sampling and Assays}

High-density lipoprotein (HDL) cholesterol, triglycerides (L Type Wako Triglyceride $\cdot \mathrm{H}$, Wako Chemical, Osaka, Japan) and blood glucose (hexokinase method) were measured at the Okayama Southern Institute of Health, Okayama Health Foundation. The accuracy of the measurements was maintained during the study period.

\subsection{Definition of Metabolic Syndrome}

The syndrome was defined [1], among men with an abdominal circumference in excess of $85 \mathrm{~cm}$ and women with an abdominal circumference in excess of $90 \mathrm{~cm} \mathrm{[12],}$ as having two or more of the following components: 1) dyslipidemia: triglyceride $\geq 150 \mathrm{mg} / \mathrm{dl}$ and/or HDL cholesterol $<40 \mathrm{mg} / \mathrm{dl}$; 2) hypertension: blood pressure $\geq$
130/85 mmHg; 3) Impaired glucose tolerance: fasting blood glucose $\geq 110 \mathrm{mg} / \mathrm{dl}$.

\subsection{Coffee, Tea and Green Tea Consumption}

Subjects were asked how many cups of coffee, tea and green tea per week. The way of drinking was not asked.

\subsection{Exercise Habits}

The data on exercise habits were obtained at interviews conducted by well-trained staff using the structured method of the National Nutrition Survey in Japan. The subjects were asked if they currently exercise (over 30 min per session, 2 times per week for duration of 3 months). When the answer was "yes", they were classified as subjects with exercise habits. When the answer was "no", they were classified as subjects without exercise habits.

\subsection{Smoking Habits}

The data on cigarette smoking was obtained at interviews by well-trained staff in a structured way. The subjects were asked if they currently smoked cigarettes. When the answer was "yes", they were classified as current smokers. When the answer was "no", they were classified as non-smokers.

\subsection{Statistical Analysis}

Data are expressed as means \pm standard deviation (SD) 
values. It is well known that the difference of prevalence of men and women in Japanese [2]. Therefore, we analyzed separately in men and women. A comparison of parameters between the two groups was made using the unpaired $t$-test, covariance analysis and logistic regression analysis. Simple correlation analysis was performed as well to test for the significance of the linear relationship among continuous variables: $p<0.05$ was considered statistically significant.

\section{RESULTS}

Clinical profiles are summarized in Table 1. The mean coffee consumption was $10.1 \pm 9.1 \mathrm{cups} /$ week/person in men and $8.6 \pm 9.6$ cups/week/person in women. The mean tea consumption was $1.5 \pm 4.1 \mathrm{cups} /$ week/person in men and $2.1 \pm 2.9 \mathrm{cups} / \mathrm{week} / \mathrm{person}$ in women. The mean green tea consumption was $6.3 \pm 6.6 \mathrm{cups} / \mathrm{week} /$ person in men and $7.4 \pm 8.6 \mathrm{cups} /$ week/person in women, respectively.

We evaluated the relationship between coffee, tea and green tea consumption and age (Table 2). Coffee consumption was weakly and positively correlated with age in both sexes. Green tea consumption in women was also weakly and positively correlated with age.

A total of 34 men $(22.7 \%)$ and 10 women (4.4\%) were diagnosed with metabolic syndrome in subjects without medications. In subjects not taking medications, we compared coffee, tea and green tea consumption between the groups with and without each component of the Japanese definition of metabolic syndrome (Table 3). To avoid the influence of age, we used age as a covariate and compared coffee, tea and green tea consumption between Japanese with and those without metabolic syndrome components using covariance analysis. Tea consumption in men with dyslipidemia and coffee consumption in women with abdominal obesity and hypertension were significantly higher than those in subjects without, even after adjusting for age. Coffee consumption in women with dyslipidemia was significantly lower than that in

Table 2. Relationship between coffee, tea and green tea consumption and age.

\begin{tabular}{lcc}
\hline & $\mathrm{r}$ & $p$ \\
\hline \multicolumn{1}{c}{ Men } & & \\
Coffee consumption (cup/week) & 0.169 & $\mathbf{0 . 0 3 9 2}$ \\
Tea consumption (cup/week) & -0.025 & 0.7613 \\
Green tea consumption (cup/week) & 0.146 & 0.0738 \\
$\quad$ Women & & \\
Coffee consumption (cup/week) & 0.378 & $<\mathbf{0 . 0 0 0 1}$ \\
Tea consumption (cup/week) & 0.045 & 0.5001 \\
Green tea consumption (cup/week) & 0.334 & $<\mathbf{0 . 0 0 0 1}$ \\
\hline
\end{tabular}

subjects without dyslipidemia.

Finally, we evaluated the coffee, tea and green tea consumptions with and without metabolic syndrome using logistic regression analysis. There was significant difference of green tea consumption between men with and without metabolic syndrome after adjusting for age, exercise habits, smoking habits and coffee, tea consumptions. There were also significant differences of coffee, and green tea consumptions between women with and without metabolic syndrome after various parameters (Table 4).

\section{DISCUSSION}

We firstly evaluated the link between metabolic syndrome using developed in Japan and coffee, tea and green tea consumption in Japanese without taking any medications. The clear differences of these consumptions were not noted between Japanese with and without metabolic components in this cross-sectional study.

Hino et al. reported that they examined the relationship between metabolic syndrome using Japanese criteria and the consumption of coffee or green tea in 1902 Japanese over 40 . They concluded that coffee but not green tea consumption was inversely associated with metabolic syndrome [13]. In turn, Balk et al. showed in prospective study that coffee consumption was signifycantly associated with lower HDL cholesterol in women and coffee consumption was not associated with any of the components of the metabolic syndrome in men [14]. Driessen et al. also showed that coffee consumption was not associated with metabolic syndrome and its components in healthy samples in 174 men and 194 women followed up for 9 years [15]. Therefore, the link between coffee, tea and green tea consumption and metabolic syndrome was not clearly proved. In this study, we compared coffee, tea and green tea consumption between subjects with and without metabolic components. Significant differences of some consumption between subjects with and without metabolic syndrome components were noted after adjusting for age. In addition, there were also some significant differences of coffee and green tea consumption between subjects with and without metabolic syndrome after adjusting for various parameters. However, we could not also prove the clear link between metabolic syndrome and its components and coffee, tea and green tea consumption in Japanese without medications.

Potential limitations remain in this study. First, our study was a cross sectional and not a longitudinal study. Second, 150 men and 227 women, all of whom wanted to change their lifestyle, underwent measurements for this study: they were therefore more health-conscious than the average person. Third, we could not clarify the mechanism the link between metabolic syndrome and 
Table 3. Comparison of coffee, tea and green tea consumption between subjects with and without metabolic components.

\begin{tabular}{|c|c|c|c|c|}
\hline & & & $p$ & $p$ (After adjusting for age) \\
\hline Men & Abdominal obesity (-) & Abdominal obesity $(+)$ & & \\
\hline Number of subjects & 63 & 87 & & \\
\hline Age & $45.0 \pm 13.2$ & $47.9 \pm 11.6$ & 0.1609 & \\
\hline Coffee consumption (cup/week) & $10.7 \pm 9.8$ & $9.8 \pm 8.5$ & 0.5513 & 0.6662 \\
\hline Tea consumption (cup/week) & $1.9 \pm 5.3$ & $1.1 \pm 2.8$ & 0.2351 & 0.5626 \\
\hline \multirow[t]{2}{*}{ Green tea consumption (cup/week) } & $6.0 \pm 6.0$ & $6.5 \pm 7.0$ & 0.6713 & 0.2377 \\
\hline & Dyslipidemia (-) & Dyslipidemia $(+)$ & & \\
\hline Number of subjects & 98 & 52 & & \\
\hline Age & $45.8 \pm 13.6$ & $48.4 \pm 9.5$ & 0.2118 & \\
\hline Coffee consumption (cup/week) & $8.9 \pm 8.0$ & $12.4 \pm 10.5$ & 0.0244 & 0.6096 \\
\hline Tea consumption (cup/week) & $1.4 \pm 3.7$ & $1.6 \pm 4.7$ & 0.8535 & 0.0238 \\
\hline \multirow[t]{2}{*}{ Green tea consumption (cup/week) } & $6.5 \pm 6.9$ & $5.8 \pm 6.2$ & 0.5203 & 0.6581 \\
\hline & Hypertension (-) & Hypertension $(+)$ & & \\
\hline Number of subjects & 80 & 70 & & \\
\hline Age & $45.7 \pm 13.4$ & $47.9 \pm 11.0$ & 0.2801 & \\
\hline Coffee consumption (cup/week) & $9.7 \pm 8.5$ & $10.7 \pm 9.7$ & 0.4971 & 0.2058 \\
\hline Tea consumption (cup/week) & $1.1 \pm 2.7$ & $1.9 \pm 5.1$ & 0.2620 & 0.9057 \\
\hline \multirow[t]{2}{*}{ Green tea consumption (cup/week) } & $6.5 \pm 6.0$ & $6.1 \pm 7.3$ & 0.7292 & 0.1660 \\
\hline & Inpaired glucose tolerance $(-)$ & Inpaired glucose tolerance $(+)$ & & \\
\hline Number of subjects & 131 & 19 & & \\
\hline Age & $46.0 \pm 12.7$ & $51.2 \pm 8.8$ & 0.0913 & \\
\hline Coffee consumption (cup/week) & $10.2 \pm 9.3$ & $9.9 \pm 7.1$ & 0.9186 & 0.3668 \\
\hline Tea consumption (cup/week) & $1.2 \pm 3.4$ & $3.1 \pm 7.1$ & 0.0602 & 0.0810 \\
\hline Green tea consumption (cup/week) & $6.5 \pm 6.8$ & $5.1 \pm 4.7$ & 0.4069 & 0.8693 \\
\hline Women & Abdominal obesity (-) & Abdominal obesity $(+)$ & & \\
\hline Number of subjects & 198 & 29 & & \\
\hline Age & $42.6 \pm 14.8$ & $49.3 \pm 13.7$ & 0.0243 & \\
\hline Coffee consumption (cup/week) & $8.5 \pm 8.5$ & $9.7 \pm 11.8$ & 0.4906 & 0.0009 \\
\hline Tea consumption (cup/week) & $2.2 \pm 3.0$ & $1.9 \pm 2.7$ & 0.7013 & 0.4672 \\
\hline \multirow[t]{2}{*}{ Green tea consumption (cup/week) } & $7.3 \pm 7.7$ & $8.0 \pm 13.6$ & 0.6877 & 0.5246 \\
\hline & Dyslipidemia (-) & Dyslipidemia $(+)$ & & \\
\hline Number of subjects & 199 & 28 & & \\
\hline Age & $43.0 \pm 14.5$ & $47.3 \pm 16.9$ & 0.1449 & \\
\hline Coffee consumption (cup/week) & $8.6 \pm 8.8$ & $8.6 \pm 10.6$ & 0.9795 & 0.0274 \\
\hline Tea consumption (cup/week) & $2.1 \pm 2.9$ & $2.1 \pm 3.3$ & 0.9686 & 0.2693 \\
\hline \multirow[t]{2}{*}{ Green tea consumption (cup/week) } & $7.6 \pm 8.9$ & $6.0 \pm 6.2$ & 0.3611 & 0.8384 \\
\hline & Hypertension (-) & Hypertension $(+)$ & & \\
\hline Number of subjects & 185 & 42 & & \\
\hline Age & $40.6 \pm 14.2$ & $56.4 \pm 10.1$ & $<0.0001$ & \\
\hline Coffee consumption (cup/week) & $8.4 \pm 9.1$ & $9.7 \pm 8.4$ & 0.3902 & 0.0163 \\
\hline Tea consumption (cup/week) & $2.2 \pm 3.1$ & $1.8 \pm 2.2$ & 0.4745 & 0.7635 \\
\hline \multirow[t]{2}{*}{ Green tea consumption (cup/week) } & $7.3 \pm 8.8$ & $8.1 \pm 7.7$ & 0.5953 & 0.6948 \\
\hline & Inpaired glucose tolerance $(-)$ & Inpaired glucose tolerance $(+)$ & & \\
\hline Number of subjects & 216 & 11 & & \\
\hline Age & $43.1 \pm 14.6$ & $50.9 \pm 17.8$ & 0.0892 & \\
\hline Coffee consumption (cup/week) & $8.6 \pm 9.0$ & $9.1 \pm 9.7$ & 0.8568 & 0.8598 \\
\hline Tea consumption (cup/week) & $2.2 \pm 3.0$ & $1.5 \pm 2.2$ & 0.4386 & 0.6263 \\
\hline Green tea consumption (cup/week) & $7.4 \pm 8.6$ & $8.5 \pm 8.5$ & 0.6614 & 0.5721 \\
\hline
\end{tabular}


Table 4. Comparison of coffee, tea and green tea consumption between subjects with and without metabolic components.

\begin{tabular}{|c|c|c|c|c|c|}
\hline & Meabolic syndrome (-) & Metabolic syndrome $(+)$ & $p$ & $p$ (After adjusting for age) & $p$ \\
\hline \multicolumn{6}{|l|}{ Men } \\
\hline Number of subjects & 116 & 34 & & & \\
\hline Age & $46.0 \pm 13.0$ & $48.9 \pm 9.8$ & 0.2279 & & \\
\hline Coffee consumption (cup/week) & $9.6 \pm 9.1$ & $12.0 \pm 8.7$ & 0.1756 & 0.9010 & $0.6155^{*}$ \\
\hline Tea consumption (cup/week) & $1.7 \pm 4.5$ & $0.8 \pm 2.0$ & 0.2478 & 0.3793 & $0.2520^{* *}$ \\
\hline Green tea consumption (cup/week) & $6.8 \pm 6.9$ & $4.5 \pm 5.4$ & 0.0783 & 0.8514 & $0.0072^{* * *}$ \\
\hline \multicolumn{6}{|l|}{ Women } \\
\hline Number of subjects & 217 & 10 & & & \\
\hline Age & $42.8 \pm 14.7$ & $59.3 \pm 9.5$ & 0.0005 & & \\
\hline Coffee consumption (cup/week) & $8.7 \pm 9.1$ & $6.1 \pm 5.0$ & 0.3670 & 0.4577 & $0.0312^{*}$ \\
\hline Tea consumption (cup/week) & $2.2 \pm 3.0$ & $1.3 \pm 2.1$ & 0.3649 & 0.8545 & $0.3780^{* *}$ \\
\hline Green tea consumption (cup/week) & $7.5 \pm 8.7$ & $4.9 \pm 4.6$ & 0.3432 & 0.9777 & $0.0208^{* * *}$ \\
\hline
\end{tabular}

coffee, tea and green tea consumption. Some studies have showed that habitual coffee consumption may improve insulin resistance and abdominal glucose metabolism [9-11]. However, we evaluated simplistic scientific and speculative approach to study these subjects without any laboratory measurements i.e. adipokines and insulin resistance. In addition, small sample size (low statistical power) in subjects with metabolic syndrome, especially women, and possibility of misclassification relying only on purported consumption made it difficult to prove the link. Fourth, for, example, the coffee consumption was reported to be $10.6 \mathrm{cups} / \mathrm{week} /$ person in 2008 , and it is the highest between 40 and 59 (men: 13.5 cups/week/ person, women: 14.2 cups/week/person) in Japanese by All Japan Coffee Association [7]. The coffee consumption was gradually increasing [7]. In this study, the mean of the coffee consumption was lower than that in the previous report. Further prospective studies are needed in Japanese subjects in Japanese.

\section{REFERENCES}

[1] Japanese Society of Internal Medicine (2005) Definition and the diagnostic standard for metabolic syndromeCommittee to evaluate diagnostic standards for metabolic syndrome. Nippon Naika Gakkai Zasshi, 94, 794-809. doi:10.2169/naika.94.794

[2] Miyatake, N., Kawasaki, Y., Nishikawa, H., Takenami, S. and Numata, T. (2006) Prevalence of metabolic syndrome in Okayama prefecture. Internal Medicine, 45, 107-108. doi:10.2169/internalmedicine.45.1509

[3] Wilson, P.W., Kannel, W.B., Silbershatz, H. and D'Agostino, R.B. (1999) Clustering of metabolic factors and coronary heart disease. Archives of Internal Medicine, 159, 1104-1109. doi:10.1001/archinte.159.10.1104
[4] Miyatake, N., Wada, J., Kawasaki, Y., Matsumoto, S., Makino, H. and Numata, T. (2006) Relationship between metabolic syndrome and proteinuria in the Japanese population. Internal Medicine, 45, 599-603. doi:10.2169/internalmedicine.45.1681

[5] Miyatake, N., Matsumoto, S., Makino, H. and Numata, T. (2007) Comparison of hepatic enzymes between Japanese men with and without metabolic syndrome. Acta Medica Okayama, 61, 31-34.

[6] Numata, T., Miyatake, N., Wada, J. and Makino, H. (2008) Comparison of serum uric acid levels between Japanese with and without metabolic syndrome. Diabetes Research and Clinical Practice, 80, 1-5. doi:10.1016/j.diabres.2007.10.031

[7] http://coffee.ajca.or.jp/wp-content/uploads/2011/09/data0 4_2011-04.pdf (in Japanese).

[8] Iso, H., Date, C., Wakai, K., Fukui, M. and Tamakoshi, A. (2006) The relationship between green tea and total caffeine intake and risk for self-reported type 2 diabetes among Japanese adults. Annals of Internal Medicine, 144, 554-562.

[9] Tunnicliffe, J.M. and Shearer, J. (2008) Coffee, glucose homeostasis, and insulin resistance: Physiological mechanisms and mediators. Applied Physiology, Nutrition and Metabolism, 33, 1290-1300. doi:10.1139/H08-123

[10] Van Dam, R.M. (2008) Coffee consumption and risk of type 2 diabetes, cardiovascular diseases, and cancer. Applied Physiology, Nutrition and Metabolism, 33, 12691283. doi: $10.1139 / \mathrm{H} 08-120$

[11] Greenberg, J.A., Boozer, C.N. and Geliebter, A. (2006) Coffee, diabetes, and weight control. American Journal of Clinical Nutrition, 84, 682-693.

[12] Yuji, M. (2002) New criteria for "obesity disease" in Japan: The examination committee of criteria for "obesity disease" in Japan, Japan society for the study of obesity. Circulation Journal, 66, 987-992.

[13] Hino, A., Adachi, H., Enomoto, M., Furuki, K., Shigetoh, 
Y., Ohtsuka, M., Kumagae, S., Hirai, Y., Jalaldin, A., Satoh, A. and Imaizumi, T. (2007) Habitual coffee but not green tea consumption is inversely associated with metabolic syndrome: An epidemiological study in a general Japanese population. Diabetes Research and Clinical Practice, 76, 383-389. doi:10.1016/j.diabres.2006.09.033

[14] Balk, L., Hoekstra, T. and Twisk, J. (2009) Relationship between long-term coffee consumption and components of the metabolic syndrome: The Amsterdam Growth and
Health Longitudinal Study. European Journal of Epidemiology, 24, 203-209. doi:10.1007/s10654-009-9323-1

[15] Driessen, M.T., Koppes, L.L., Veldhuis, L., Samoocha, D. and Twisk, J.W. (2009) Coffee consumption is not related to the metabolic syndrome at the age of 36 years: The Amsterdam Growth and Health Longitudinal Study. European Journal of Clinical Nutrition, 63, 536-542.

doi:10.1038/ejen.2008.6 